\title{
On density modulo 1 of some expressions containing algebraic integers
}

\author{
by \\ Roman URBan (Wrocław)
}

1. Introduction. It is a very well known result in the theory of distribution modulo 1 that for every irrational $\xi$ the sequence $\{n \xi: n \in \mathbb{N}\}$ is dense modulo 1 (and even uniformly distributed modulo 1) [13].

Let $S$ be a multiplicative semigroup of integers. The semigroup $S$ is said to be lacunary if the members $\{s \in S: s>0\}$ are of the form $s_{0}^{k}, k \in \mathbb{N}$, $s_{0} \in \mathbb{N}^{*}$. Otherwise, $S$ is non-lacunary. In 1967, in his seminal paper [7], Furstenberg proved that if $S$ is a non-lacunary semigroup of integers and $\xi$ is an irrational number, then the orbit $S \xi$ is dense modulo 1 . In other words, we have the following

THEOREM 1.1 (Furstenberg, [7]). If $p, q>1$ are rationally independent integers (i.e., they are not both integer powers of the same integer) then for every irrational $\xi$ the set

$$
\left\{p^{n} q^{m} \xi: n, m \in \mathbb{N}\right\}
$$

is dense modulo 1.

In particular, the result of Furstenberg can be considered as a generalization of a theorem of Hardy and Littlewood [10], which asserts that if $r$ is a positive integer and $\xi$ is an irrational number, then the set $\left\{q^{r} \xi: q \in \mathbb{N}\right\}$ is dense modulo 1. Furstenberg's proof is based on a fundamental idea of disjointness of dynamical systems, which he introduced in the same paper [7] (see also [17], where Furstenberg's original proof is outlined). In 1994 Boshernitzan gave an elementary proof of Furstenberg's theorem in [6].

2000 Mathematics Subject Classification: 11J71, 54H20.

Key words and phrases: distribution modulo 1, algebraic integers, topological dynamics, semigroup of endomorphisms, ID-semigroup, invariant sets.

Research supported in part by RTN Harmonic Analysis and Related Problems contract HPRN-CT-2001-00273-HARP, the European Commission Marie Curie Host Fellowship for the Transfer of Knowledge "Harmonic Analysis, Nonlinear Analysis and Probability" MTKD-CT-2004-013389 and by the MNiSW research grant N201 012 31/1020. 
One possible direction of generalizations is to consider $p$ and $q$ in Theorem 1.1 not necessarily integer. This was done by Berend in [4]. Let us state his result precisely.

Let $K$ be a real algebraic number field (i.e., a finite extension of $\mathbb{Q}$ ) and $S$ a subsemigroup of its multiplicative group $K^{*}$. According to [4], the semigroup $S$ is said to be $\mathrm{DM}_{1}$ if $S \xi$ is dense modulo 1 for every $\xi \neq 0$, and almost $\mathrm{DM}_{1}$ if $S \xi$ is dense modulo 1 for every $\xi \notin K$. We say that two numbers $\lambda$ and $\mu$ are rationally dependent if there are integers $m$ and $n$, not both 0 , such that $\lambda^{m}=\mu^{n}$, and rationally independent otherwise. The semigroup $S$ is said to be one-parameter if all its elements are integer powers of a single number; weakly one-parameter if any two of its elements are rationally dependent, and multi-parameter otherwise. If $[K: \mathbb{Q}]=m$, we denote by $\operatorname{PS}(K)$ the semigroup consisting of all Pisot or Salem numbers of degree $m$ (see Section 2 for the definition). For a subset $A \subset K$, we denote by $\mathbb{Q}(A)$ the subfield of $K$ obtained by adjoining $A$ to $\mathbb{Q}$. Then we have the following

THEOREM 1.3 (Berend, [4]). Let $K$ be a real algebraic number field and $S$ a multi-parameter subsemigroup of $K^{*} \cap[-1,1]^{\mathrm{c}}$ with $\mathbb{Q}(S)=K$. Then $S$ is almost $\mathrm{DM}_{1}$. If , moreover, $S \not \subset \mathrm{PS}(K)$, then $S$ is $\mathrm{DM}_{1}$.

An interesting generalization of Furstenberg's result is the following

TheOREM 1.4 (Kra, [12]). Suppose that the pairs $p_{i}, q_{i} \in \mathbb{N}$, with $1<$ $p_{i}<q_{i}$ for $i=1, \ldots, k, k \in \mathbb{N},\left(p_{i}, q_{i}\right) \neq\left(p_{j}, q_{j}\right)$ for $i \neq j$, and $p_{1} \leq \cdots \leq p_{k}$, are rationally independent. Then for distinct $\xi_{1}, \ldots, \xi_{k} \in[0,1]$ with at least one $\xi_{i} \notin \mathbb{Q}$ the set

is dense modulo 1 .

$$
\left\{\sum_{i=1}^{k} p_{i}^{n} q_{i}^{m} \xi_{i}: n, m \in \mathbb{N}\right\}
$$

(See the paper of Meiri [14] for an alternative proof of part of Kra's result via measure-theoretic methods.)

Inspired by Kra's theorem we state the following conjecture generalizing Berend's Theorem 1.3.

Conjecture 1.5. Let $k \in \mathbb{N}$ be fixed, and let $\lambda_{i}, \mu_{i}$, for $1 \leq i \leq k$, be real algebraic numbers with absolute values greater than 1 . Assume that, for $i=1, \ldots, k$, the pairs $\lambda_{i}, \mu_{i}$ are rationally independent, and $\left(\lambda_{i}, \mu_{i}\right) \neq$ $\left(\lambda_{j}, \mu_{j}\right)$ for $i \neq j$. Then for any real numbers $\xi_{1}, \ldots, \xi_{k}$ with at least one $\xi_{i} \notin \mathbb{Q}\left(\bigcup_{i=1}^{k}\left\{\lambda_{i}, \mu_{i}\right\}\right)$ the set

is dense modulo 1.

$$
\left\{\sum_{i=1}^{k} \lambda_{i}^{n} \mu_{i}^{m} \xi_{i}: n, m \in \mathbb{N}\right\}
$$


The aim of this paper is to make a first step toward a proof of Conjecture 1.5. Namely, using some topological dynamics methods in the spirit of Berend [4] and Kra [12], we prove the following

THEOREM 1.6. Let $\lambda_{1}, \mu_{1}$ and $\lambda_{2}, \mu_{2}$ be two distinct pairs of rationally independent real algebraic integers of degree 2 , with absolute values greater than 1 , such that the absolute values of their conjugates $\widetilde{\lambda}_{1}, \widetilde{\mu}_{1}, \widetilde{\lambda}_{2}, \widetilde{\mu}_{2}$ are also greater than 1. Let

$$
\begin{array}{ll}
\mu_{1}=g_{1}\left(\lambda_{1}\right) & \text { for some } g_{1} \in \mathbb{Z}[x], \\
\mu_{2}=g_{2}\left(\lambda_{2}\right) & \text { for some } g_{2} \in \mathbb{Z}[x] .
\end{array}
$$

Assume that at least one element in each pair $\lambda_{i}, \mu_{i}$ has all non-negative powers irrational. Assume further that there exist $k, l, k^{\prime}, l^{\prime} \in \mathbb{N}$ such that

$$
\min \left\{\left|\lambda_{2}\right|^{k}\left|\mu_{2}\right|^{l},\left|\widetilde{\lambda}_{2}\right|^{k}\left|\widetilde{\mu}_{2}\right|^{l}\right\}>\max \left\{\left|\lambda_{1}\right|^{k}\left|\mu_{1}\right|^{l},\left|\widetilde{\lambda}_{1}\right|^{k}\left|\widetilde{\mu}_{1}\right|^{l}\right\}
$$

and

$$
\min \left\{\left|\lambda_{1}\right|^{k^{\prime}}\left|\mu_{1}\right|^{l^{\prime}},\left|\widetilde{\lambda}_{1}\right|^{k^{\prime}}\left|\widetilde{\mu}_{1}\right|^{l^{\prime}}\right\}>\max \left\{\left|\lambda_{2}\right|^{k^{\prime}}\left|\mu_{2}\right|^{l^{\prime}},\left|\widetilde{\lambda}_{2}\right|^{k^{\prime}}\left|\widetilde{\mu}_{2}\right|^{l^{\prime}}\right\} .
$$

Then for any real numbers $\xi_{1}, \xi_{2}$ with at least one $\xi_{i} \neq 0$ the set

$$
\left\{\lambda_{1}^{n} \mu_{1}^{m} \xi_{1}+\lambda_{2}^{n} \mu_{2}^{m} \xi_{2}: n, m \in \mathbb{N}\right\}
$$

is dense modulo 1.

To have in mind a simple example illustrating Theorem 1.6 consider the following expression:

$$
(\sqrt{23}+1)^{n}(\sqrt{23}+2)^{m} \xi_{1}+(\sqrt{61}+1)^{n}(\sqrt{61}-6)^{m} \xi_{2} .
$$

It is easy to verify that the density modulo 1 of the expressions of the form (1.10) with $m, n \in \mathbb{N}$ follows from Theorem 1.6, provided that at least one of the $\xi_{i}$ 's is non-zero. Actually, (1.10) is a special case of a general situation when assumptions (1.7) and (1.8) of Theorem 1.6 hold, namely, when

$$
\left|\lambda_{2}\right|>\left|\widetilde{\lambda}_{2}\right|>\left|\lambda_{1}\right|>\left|\widetilde{\lambda}_{1}\right|>1 \text { and }\left|\mu_{1}\right|>\left|\widetilde{\mu}_{1}\right|>\left|\mu_{2}\right|>\left|\widetilde{\mu}_{2}\right|>1 \text {. }
$$

It is easy to check that (1.11) implies (1.7) and (1.8).

As a corollary from the proof of Theorem 1.6 we will get the following strengthening of Theorem 1.6 which gives density modulo 1 of (1.9) in the case when not all of $\lambda_{i}, \mu_{i}$ are of degree 2 :

Corollary 1.12. Let $\lambda_{1}, \mu_{1}$ and $\lambda_{2}, \mu_{2}$ be two distinct pairs of rationally independent real algebraic integers of degree 1 or 2 , with absolute values greater than 1 , such that the absolute values of their conjugates $\widetilde{\lambda}_{1}, \widetilde{\mu}_{1}, \widetilde{\lambda}_{2}, \widetilde{\mu}_{2}$ are also greater than 1 (for an algebraic integer $\lambda$ of degree 1 we define $\widetilde{\lambda}$ to be $\lambda$ ). Assume that for each $i$, if $\lambda_{i}$ or $\mu_{i}$ has degree 2 , then at least one of them has all non-negative powers irrational. Assume further that if $\lambda_{i}$ and $\mu_{i}$ 
are both of degree 2 then

$$
\mu_{i}=g_{i}\left(\lambda_{i}\right) \quad \text { for some } g_{i} \in \mathbb{Z}[x] \text {. }
$$

Assume also that there exist $k, l, k^{\prime}, l^{\prime} \in \mathbb{N}$ such that (1.7) and (1.8) hold, and $\xi_{i}$ is irrational if $\lambda_{i}, \mu_{i} \in \mathbb{Q}$. Then the conclusion of Theorem 1.6 holds.

As an example illustrating the above corollary consider

$$
(3+\sqrt{3})^{n} 2^{m}+5^{n} 7^{m} \xi_{2} \sqrt{2} .
$$

Another kind of generalization of Furstenberg's Theorem 1.1, which we are going to use in the proof of our result, is to consider higher-dimensional analogues. Notice that, in terms of dynamical systems, Furstenberg's theorem says that the orbits of the semigroup generated by $p$ and $q$ and acting on $\mathbb{T}=\mathbb{R} / \mathbb{Z}$ are finite or dense, or equivalently (see [9] for details), the only infinite closed $p$ - and $q$-invariant subset of $\mathbb{T}=\mathbb{R} / \mathbb{Z}$ is $\mathbb{T}$ itself. Clearly, there are many closed infinite $p$-invariant (or $q$-invariant) proper subsets of $\mathbb{T}$. Hence, Furstenberg's theorem gives a remarkable rigidity property of the joint $p$ - and $q$-action on the one-dimensional torus.

A generalization of this rigidity property to a commutative semigroup of non-singular $d \times d$ matrices with integer coefficients acting by endomorphisms on the $d$-dimensional torus $\mathbb{T}^{d}=\mathbb{R}^{d} / \mathbb{Z}^{d}$, and to the action of commutative semigroups of endomorphisms acting on other compact abelian groups, was given by Berend in [2] and [3], respectively. Recently some generalizations for non-commutative semigroups of endomorphisms acting on $\mathbb{T}^{d}$ have been obtained in $[8,9,16]$.

The structure of the paper is as follows. In Section 2 we recall some elementary definitions. Then in Section 3, following Berend [2, 3], we recall the definition of an ID-semigroup of endomorphisms of the $d$-dimensional torus $\mathbb{T}^{d}$ and state Berend's theorem, [2], which gives conditions that guarantee that a given semigroup of endomorphisms of $\mathbb{T}^{d}$ is an ID-semigroup. In Section 4 we consider two commutative semigroups $\Sigma_{1}$ and $\Sigma_{2}$ of endomorphisms of the 2-dimensional torus and study the closed invariant sets for the corresponding action of $\Sigma_{1} \times \Sigma_{2}$ on the product $\mathbb{T}^{2} \times \mathbb{T}^{2}$. Finally in Section 5 we prove the main result of the paper.

Acknowledgements. The author is grateful to Yves Guivarc'h for his helpful remarks concerning the content of this paper. The author also wishes to thank the referee for a series of essential remarks that improved the overall presentation of the result.

2. Some definitions. We say that $P \in \mathbb{Z}[x]$ is monic if the leading coefficient of $P$ is one, and reduced if its coefficients are relatively prime. A real algebraic integer is any real root of a monic polynomial $P \in \mathbb{Z}[x]$, whereas an algebraic number is any root (real or complex) of a (not necessarily monic) 
non-constant polynomial $P \in \mathbb{Z}[x]$. The minimal polynomial of an algebraic number $\theta$ is the reduced element $Q$ of $\mathbb{Z}[x]$ of the least degree such that $Q(\theta)=0$. If $\theta$ is an algebraic number, the roots of its minimal polynomial are simple. The degree of an algebraic number is the degree of its minimal polynomial.

Let $\theta$ be an algebraic integer of degree $n$ and let $P \in \mathbb{Z}[x]$ be the minimal polynomial of $\theta$. The $n-1$ other distinct (real or complex) roots $\theta_{2}, \ldots, \theta_{n}$ of $P$ are called conjugates of $\theta$.

A Pisot number is a real algebraic integer $\theta$ greater than 1 whose conjugates $\theta_{2}, \ldots, \theta_{n}$ satisfy the inequalities $\left|\theta_{2}\right|<1, \ldots,\left|\theta_{n}\right|<1$.

A Salem number is a real algebraic integer $\theta$ greater than 1 whose conjugates $\theta_{2}, \ldots, \theta_{n}$ satisfy the inequalities $\left|\theta_{j}\right| \leq 1,2 \leq j \leq n$, with equality for at least one $j$.

If $\theta$ is a Salem number of degree $n$, then $n$ is an even integer, $n=2 m$, and the conjugates of $\theta$ are $1 / \theta$ and $n-2$ pairwise-conjugate complex numbers of absolute value $1,\left(\zeta_{1}, \bar{\zeta}_{1}\right), \ldots,\left(\zeta_{m-1}, \bar{\zeta}_{m-1}\right)$ (see [15]).

For more about Pisot and Salem numbers see [15, 5].

3. ID-semigroups of endomorphisms acting on $\mathbb{T}^{d}$. Following [2, 3], we say that the semigroup $\Sigma$ of continuous endomorphisms of a compact group $G$ has the ID-property (or simply that $\Sigma$ is an ID-semigroup) if the only infinite closed $\Sigma$-invariant subset of $G$ is $G$ itself. (ID-property stands for infinite invariant is dense.)

Berend in [2] gave necessary and sufficient conditions in arithmetical terms for a commutative semigroup $\Sigma$ of endomorphisms of the $r$-dimensional torus $\mathbb{T}^{d}=\mathbb{R}^{d} / \mathbb{Z}^{d}$ to have the ID-property. Namely, he proved the following.

TheOREM 3.1 (Berend, [2, Theorem 2.1]). A commutative semigroup $\Sigma$ of endomorphisms of $\mathbb{T}^{d}$ has the ID-property if and only if the following hold:

(i) There exists an endomorphism $\sigma \in \Sigma$ such that the characteristic polynomial $f_{\sigma^{n}}$ of $\sigma^{n}$ is irreducible over $\mathbb{Z}$ for every positive integer $n$.

(ii) For every common eigenvector $v$ of $\Sigma$ there exists an endomorphism $\sigma_{v} \in \Sigma$ whose eigenvalue in the direction of $v$ is of norm greater than 1 .

(iii) $\Sigma$ contains a pair of rationally independent endomorphisms.

We say, exactly as in the case of real numbers, that two endomorphisms $\sigma$ and $\tau$ are rationally dependent if there are integers $m$ and $n$, not both 0 , such that $\sigma^{m}=\tau^{n}$, and rationally independent otherwise. 
REMARK 3.2. Let $\Sigma$ be a commutative ID-semigroup of endomorphisms of $\mathbb{T}^{d}$. Then the $\Sigma$-orbit of the point $x \in \mathbb{T}^{d}$ is finite if and only if $x$ is a rational element, i.e., $x=r / q, r \in \mathbb{Z}^{d}, q \in \mathbb{N}$ (see [2]).

4. A semigroup $M$ acting on the product $\mathbb{T}^{2} \times \mathbb{T}^{2}$. Let $\sigma_{1}, \tau_{1}$ and $\sigma_{2}, \tau_{2}$ be two pairs of rationally independent and commuting endomorphisms of $\mathbb{T}^{2}$ (given by non-singular $2 \times 2$-matrices with integer coefficients). We assume that for $i=1,2$ the semigroups $\Sigma_{i}=\left\langle\sigma_{i}, \tau_{i}\right\rangle$ generated by $\sigma_{i}$ and $\tau_{i}$ satisfy the conditions of Theorem 3.1. Let $M_{\sigma}=\left(\begin{array}{cc}\sigma_{1} & 0 \\ 0 & \sigma_{2}\end{array}\right)$ and $M_{\tau}=\left(\begin{array}{cc}\tau_{1} & 0 \\ 0 & \tau_{2}\end{array}\right)$. We consider the actions of $M_{\sigma}$ and $M_{\tau}$ on the product $\mathbb{T}^{2} \times \mathbb{T}^{2}$. We denote by $M$ the semigroup of endomorphisms of $\mathbb{T}^{2} \times \mathbb{T}^{2}$ generated by $M_{\sigma}$ and $M_{\tau}$.

We start with the following

Lemma 4.1. Let $\sigma$ be an invertible $d \times d$-matrix with integer entries. Let $r=x / q \in \mathbb{T}^{d}, x \in \mathbb{Z}^{d}, q \in \mathbb{N}$, be a rational element such that the denominator $q$ is relatively prime to $\operatorname{det} \sigma$. Then there exists $k \in \mathbb{N}$ such that

$$
\sigma^{k} r \equiv r\left(\bmod \mathbb{Z}^{d}\right) \text {. }
$$

Proof. We observe that $\sigma$ acts naturally on the finite set $(\mathbb{Z} / q \mathbb{Z})^{d}$. Denote by $\bar{\sigma}$ the corresponding endomorphism of the module $(\mathbb{Z} / q \mathbb{Z})^{d}$ over the finite ring $\mathbb{Z} / q \mathbb{Z}$. Thus we have an action of the semigroup $\mathbb{N}$ on $(\mathbb{Z} / q \mathbb{Z})^{d}$, given by $k . x=\bar{\sigma}^{k} x, k \in \mathbb{N}, x \in(\mathbb{Z} / q \mathbb{Z})^{d}$. Clearly, $\operatorname{det} \bar{\sigma}$ is the congruence class of $\operatorname{det} \sigma$ in $\mathbb{Z} / q \mathbb{Z}$. Since $q$ is relatively prime to $\operatorname{det} \sigma$, we conclude that $\operatorname{det} \bar{\sigma} \neq 0$, hence $\bar{\sigma} \in \mathrm{GL}(d, \mathbb{Z} / q \mathbb{Z})$. Thus $\left\{\bar{\sigma}^{k}: k \in \mathbb{N}\right\}$ is a semigroup contained in the finite group $\operatorname{GL}(d, \mathbb{Z} / q \mathbb{Z})$; it follows that $\left\{\bar{\sigma}^{k}: k \in \mathbb{N}\right\}$ is a group. Thus there exists $k$ such that $\bar{\sigma}^{k}=\mathrm{Id}$ and the lemma is proved.

For a given subset $A \subset \mathbb{T}^{2} \times \mathbb{T}^{2}$ and $x \in \mathbb{T}^{2}$, we define

$$
A_{x}=\left\{t \in \mathbb{T}^{2}:(t, x) \in A\right\} .
$$

The next three lemmas generalize Lemmas 3.1-3.3 from [12], where diagonal $2 \times 2$-matrices with integer entries acting on $\mathbb{T} \times \mathbb{T}$ were considered, to our higher dimensional situation. Berend's Theorem 3.1, together with Lemma 4.1 above, allows us to extend the results from [12] to the action of $M$ on $\mathbb{T}^{2} \times \mathbb{T}^{2}$. For clarity of exposition, we give detailed proofs.

Lemma 4.2. Let $A$ be a non-empty, $M_{\sigma^{-}}$and $M_{\tau}$-invariant closed subset of $\mathbb{T}^{2} \times \mathbb{T}^{2}$. Then the set $P=\left\{t \in \mathbb{T}^{2}: A_{x} \neq \emptyset\right\}$ is either the whole $\mathbb{T}^{2}$ or a finite set of rational elements in $\mathbb{T}^{2}$. Furthermore, if $q \in \mathbb{T}^{2}$ is a rational element whose denominator is relatively prime to $\operatorname{det} \sigma_{2}$ and $\operatorname{det} \tau_{2}$, then $A_{q}$ is either empty, a finite set of rational elements, or the whole $\mathbb{T}^{2}$.

Proof. Clearly, $P$ is non-empty (since $A$ is) and closed in $\mathbb{T}^{2}$. Moreover, since $A$ is $M_{\sigma^{-}}$and $M_{\tau^{-}}$invariant it follows that $P$ is $\sigma_{2^{-}}$and $\tau_{2^{-}}$invariant. Hence, by our assumption that the semigroup $\Sigma_{2}$ generated by $\sigma_{2}, \tau_{2}$ satisfies 
the conditions of Theorem 3.1, we infer that $P$ is either a finite set of rational elements, or the whole $\mathbb{T}^{2}$.

Next, given a rational element $q$ whose denominator is relatively prime to $\operatorname{det} \sigma_{2}$ and $\operatorname{det} \tau_{2}$, and with $A_{q} \neq \emptyset$, by Lemma 4.1 we can find $k_{1}, k_{2} \in \mathbb{N}$ such that $\sigma_{2}^{k_{1}} q \equiv q\left(\bmod \mathbb{Z}^{2}\right)$ and $\tau_{2}^{k_{2}} q \equiv q\left(\bmod \mathbb{Z}^{2}\right)$. Thus $A_{q}$ is a non-empty, closed, $\sigma_{2}^{k_{1}}$ - and $\tau_{2}^{k_{2}}$-invariant subset of $\mathbb{T}^{2}$. By Theorem 3.1, it is either a finite set of rational elements, or the whole $\mathbb{T}^{2}$.

Lemma 4.3. Let $A$ be a closed, $M_{\sigma^{-}}$and $M_{\tau}$-invariant subset of $\mathbb{T}^{2} \times \mathbb{T}^{2}$. If all rational elements of $A$ are isolated in $A$, then $A$ is finite.

Proof. Consider $A^{\prime}$, the set $A$ with all rational elements removed. If $A^{\prime}$ is empty there is nothing to do. If $A^{\prime}$ is non-empty, then it is also closed and

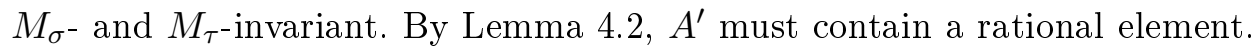
Thus $A$ is a closed set consisting of isolated points in the compact space $\mathbb{T}^{2} \times \mathbb{T}^{2}$. Hence $A$ is finite.

Lemma 4.4. Let $A$ be a closed, $M_{\sigma^{-}}$and $M_{\tau}$-invariant subset of $\mathbb{T}^{2} \times \mathbb{T}^{2}$. Let $q_{1}$ and $q_{2}$ be rational elements of $\mathbb{T}^{2}$ such that $\left(q_{1}, q_{2}\right) \in A$. Assume that the denominator of $q_{i}$ is relatively prime to $\operatorname{det} \sigma_{i}$, $\operatorname{det} \tau_{i}$ for $i=1,2$. Then there exist $n, m \in \mathbb{N}$ such that the set $A-\left(q_{1}, q_{2}\right):=\left\{\left(x-q_{1}, y-q_{2}\right):(x, y) \in\right.$ A\} is $M_{\sigma}^{n}$ - and $M_{\tau}^{m}$-invariant.

Proof. By Lemma 4.1 we can find $n, m \in \mathbb{N}$ such that

$$
\begin{array}{ll}
\sigma_{1}^{n} q_{1} \equiv q_{1}\left(\bmod \mathbb{Z}^{2}\right), & \tau_{1}^{m} q_{1} \equiv q_{1}\left(\bmod \mathbb{Z}^{2}\right), \\
\sigma_{2}^{n} q_{2} \equiv q_{2}\left(\bmod \mathbb{Z}^{2}\right), & \tau_{2}^{m} q_{2} \equiv q_{2}\left(\bmod \mathbb{Z}^{2}\right) .
\end{array}
$$

Now, the point $\left(q_{1}, q_{2}\right)$ is fixed under $M_{\sigma}^{n}$ and $M_{\tau}^{m}$. As $A$ is obviously $M_{\sigma}^{n}$ - and $M_{\tau}^{m}$-invariant, we conclude that $A-\left(q_{1}, q_{2}\right)$ is also $M_{\sigma^{-}}^{n}$ and $M_{\tau}^{m}$-invariant.

5. Proof of Theorem 1.6 and Corollary 1.12. Let $\lambda>1$ be a real algebraic integer of degree 2 with minimal (monic) polynomial $P_{\lambda} \in \mathbb{Z}[x]$,

$$
P_{\lambda}(x)=x^{2}+c_{1} x+c_{0} .
$$

With $\lambda$ we associate the companion matrix of $P_{\lambda}$,

$$
\sigma_{\lambda}=\left(\begin{array}{cc}
0 & 1 \\
-c_{0} & -c_{1}
\end{array}\right) .
$$

REMARK 5.2. We can think of $\sigma_{\lambda}$ as the matrix of multiplication by $\lambda$ in the basis of the algebraic number field $\mathbb{Q}(\lambda)$ consisting of 1 and $\lambda$, that is, if $x$ has coordinates $\alpha=\left(\alpha_{0}, \alpha_{1}\right)$ in the basis consisting of $1, \lambda$, then $\lambda x$ has coordinates $\alpha \sigma_{\lambda}$.

Let $\mu=g(\lambda)$, where $g \in \mathbb{Z}[x]$, and define the matrix $\tau_{\mu}=g\left(\sigma_{\lambda}\right)$. 
Denote by $\Sigma$ the semigroup of endomorphisms of $\mathbb{T}^{2}$ generated by $\sigma_{\lambda}$ and $\tau_{\mu}$. The vector $v=(1, \lambda)^{t}$ is an eigenvector of $\sigma_{\lambda}$ with eigenvalue $\lambda$, that is, $\sigma_{\lambda} v=\lambda v$. Since $\Sigma$ is a commutative semigroup, $v$ is a common eigenvector of $\Sigma$, in particular $\tau_{\mu} v=g\left(\sigma_{\lambda}\right) v=g(\lambda) v=\mu v$.

Let $\lambda_{1}, \mu_{1}>1$ and $\lambda_{2}, \mu_{2}>1$ be two pairs of rationally independent algebraic integers of degree 2. Moreover, assume that $\mu_{i}=g_{i}\left(\lambda_{i}\right), g_{i} \in \mathbb{Z}[x]$. We assume that the absolute values of the conjugates $\widetilde{\lambda}_{i}, \widetilde{\mu}_{i}$ of $\lambda_{i}$ and $\mu_{i}$ are also greater than one. Now, we associate with $\lambda_{i}, \mu_{i}$ the matrices $\sigma_{i}=$ $\sigma_{\lambda_{i}}, \tau_{i}=\tau_{\mu_{i}}$, as described above. For $i=1,2$, we denote by $\Sigma_{i}=\left\langle\sigma_{i}, \tau_{i}\right\rangle$ the semigroups generated by $\sigma_{i}$ and $\tau_{i}$.

Clearly, $\sigma_{1}, \tau_{1}$ and $\sigma_{2}, \tau_{2}$ are rationally independent endomorphisms of $\mathbb{T}^{2}$ and for $i=1,2$, the characteristic polynomial of $\sigma_{i}^{n}$ or $\tau_{i}^{n}$ is irreducible over $\mathbb{Z}$ for every $n \in \mathbb{N}$. Furthermore, since $\widetilde{\lambda}_{i}, \widetilde{\mu}_{i}>1$, it follows that for the semigroups $\Sigma_{i}$ condition (ii) of Theorem 3.1 is also satisfied. Thus $\Sigma_{1}$ and $\Sigma_{2}$ are ID-semigroups of endomorphisms of $\mathbb{T}^{2}$. Hence, if we define $M_{\sigma}=\left(\begin{array}{cc}\sigma_{1} & 0 \\ 0 & \sigma_{2}\end{array}\right)$ and $M_{\tau}=\left(\begin{array}{cc}\tau_{1} & 0 \\ 0 & \tau_{2}\end{array}\right)$, we can apply the results of Section 4 to the semigroup $M=\left\langle M_{\sigma}, M_{\tau}\right\rangle$ of endomorphisms of $\mathbb{T}^{2} \times \mathbb{T}^{2}$.

For $\alpha=\left(\alpha_{1}, \alpha_{2}\right) \in \mathbb{T}^{2} \times \mathbb{T}^{2}$, define

$$
X_{\alpha}=\left\{\left(\sigma_{1}^{n} \tau_{1}^{m} \alpha_{1}, \sigma_{2}^{n} \tau_{2}^{m} \alpha_{2}\right) \in \mathbb{T}^{2} \times \mathbb{T}^{2}: n, m \in \mathbb{N}\right\},
$$

which is the orbit of $\alpha$ under the action of the semigroup $M$ generated by $M_{\sigma}$ and $M_{\tau}$. Let $X_{\alpha}^{\text {ac }}$ denote the set of accumulation points of $X_{\alpha}$. Clearly, $X_{\alpha}$ and $X_{\alpha}^{\text {ac }}$ are $M$-invariant. Furthermore, $X_{\alpha}^{\text {ac }}$ is closed.

The following simple lemma will be used in the proof of the next proposition. (A more general version for $d \times d$-matrices is given in [16, Lemma 6.4].)

Lemma 5.4. Let $A$ be a real invertible $2 \times 2$-matrix with two different real eigenvalues $\eta_{1}, \eta_{2}$ such that $\left|\eta_{1}\right|>\left|\eta_{2}\right|>1$. Let $v_{n}=\left(x_{n}, y_{n}\right), x_{n} \neq 0$, $y_{n} \neq 0$, be a sequence of vectors in $\mathbb{R}^{2}$ tending to $(0,0)$. Then there exists a subsequence $v_{n_{k}}$ and an increasing subsequence $\left\{j_{n_{k}}\right\}$ of $\mathbb{N}$ such that

$$
\lim _{k \rightarrow \infty} A^{j_{n_{k}}} v_{n_{k}}=w \neq 0 \quad \text { with }\|w\| \leq 1 .
$$

Proof. We can assume that $\left\|v_{n}\right\| \leq 1$. By the assumption on the eigenvalues it follows that $A$ is an expanding map, and thus for every $n$, there exists the smallest natural number $j_{n}$ such that

$$
1 /\|A\| \leq\left\|A^{j_{n}} v_{n}\right\| \leq 1 .
$$

Hence, by compactness, we can choose a subsequence $\left\{n_{k}\right\} \subset \mathbb{N}$ such that (5.5) holds.

We will also need the following 
Lemma 5.6. Let $A: \mathbb{R}^{d} \rightarrow \mathbb{R}^{d}$ be a linear map. For every $\delta>0$ there exists a norm $\|\cdot\|_{\delta}$ in $\mathbb{R}^{d}$ such that

$$
\|A\|_{\delta}<r(A)+\delta
$$

where $r(A)$ is the spectral radius of $A$ (i.e., the maximal absolute value of an eigenvalue of $A$ ).

Proof. See Proposition 1.2.2 in [11].

Proposition 5.7. With the same assumptions as in Theorem 1.6, if $(0,0) \in X_{\alpha}^{\mathrm{ac}}$ then one of the following holds:

(1) The point $(0,0)$ is isolated in $X_{\alpha}^{\mathrm{ac}}$.

(2) The set $X_{\alpha}^{\mathrm{ac}}$ contains $\mathbb{T}^{2} \times\{0\}$ or $\{0\} \times \mathbb{T}^{2}$.

Proof. Consider a general element $m$ of the semigroup $M=\left\langle M_{\sigma}, M_{\tau}\right\rangle$,

$$
m=m(k, l)=M_{\sigma}^{k} M_{\tau}^{l}=\left(\begin{array}{cc}
\sigma_{1}^{k} \tau_{1}^{l} & 0 \\
0 & \sigma_{2}^{k} \tau_{2}^{l}
\end{array}\right) \quad \text { for some } k, l \in \mathbb{N}
$$

Denote the diagonal elements of $m$, which are non-singular $2 \times 2$-matrices, by $m_{1}$ and $m_{2}$. That is, $m_{1}=\sigma_{1}^{k} \tau_{1}^{l}$ and $m_{2}=\sigma_{2}^{k} \tau_{2}^{l}$. Let $\varrho_{1}>\varrho_{1}^{\prime}\left(\varrho_{2}>\varrho_{2}^{\prime}\right.$, resp.) denote the absolute values of the eigenvalues of $m_{1}\left(m_{2}\right.$, resp.). Since $\sigma_{i}\left(\tau_{i}\right.$, resp. $)$ has eigenvalues $\lambda_{i}, \widetilde{\lambda}_{i}\left(\mu_{i}, \widetilde{\mu}_{i}\right.$, resp. $)$, we see that

$$
\varrho_{i}=\max \left\{\left|\lambda_{i}\right|^{k}\left|\mu_{i}\right|^{l},\left|\widetilde{\lambda}_{i}\right|^{k}\left|\widetilde{\mu}_{i}\right|^{l}\right\}, \quad \varrho_{i}^{\prime}=\min \left\{\left|\lambda_{i}\right|^{k}\left|\mu_{i}\right|^{l},\left|\widetilde{\lambda}_{i}\right|^{k}\left|\widetilde{\mu}_{i}\right|^{l}\right\} .
$$

Suppose that $k$ and $l$ are fixed (we will choose them appropriately later) and consider $m=\left(\begin{array}{cc}m_{1} & 0 \\ 0 & m_{2}\end{array}\right)$ acting on $V_{1} \times V_{2}:=\mathbb{R}^{2} \times \mathbb{R}^{2}$.

It follows from Lemma 5.6 that for every $\delta>0$ there exist norms $\|\cdot\|_{i, \delta}$ in $V_{i}$ such that, for every $y \in V_{i}$,

$$
\left\|m_{i} y\right\|_{i, \delta} \leq\left(\varrho_{i}+\delta\right)\|y\|_{i, \delta}
$$

Furthermore, there exist norms $\|\cdot\|_{i}$ in $V_{i}$ such that

$$
\left\|m_{i} y\right\|_{i} \geq \varrho_{i}^{\prime}\|y\|_{i}
$$

for every $y \in V_{i}$. In fact, let $T \in \mathrm{GL}(2, \mathbb{R})$ be such that $T A T^{-1}=\left(\begin{array}{cc}\varrho_{i} & 0 \\ 0 & \varrho_{i}^{\prime}\end{array}\right)$, and define $\|y\|_{i}=\|T y\|$, where $\|y\|=\sqrt{y_{1}^{2}+y_{2}^{2}}$.

By Lemma 4.2 , we can assume that the intersection of $X_{\alpha}^{\text {ac }}$ with $\{0\} \times \mathbb{T}^{2}$ (with $\mathbb{T}^{2} \times\{0\}$, resp.) either contains finitely many rational points, or equals $\{0\} \times \mathbb{T}^{2}\left(\mathbb{T}^{2} \times\{0\}\right.$, resp. $)$. Assume that $X_{\alpha}^{\text {ac }}$ contains neither $\{0\} \times \mathbb{T}^{2}$ nor $\mathbb{T}^{2} \times\{0\}$. Then, removing finitely many rational isolated points from $\{0\} \times \mathbb{T}^{2}$ and $\mathbb{T}^{2} \times\{0\}$, by applying the matrix $\left(\begin{array}{cc}q_{1} \text { Id } & 0 \\ 0 & q_{2} \text { Id }\end{array}\right)$ to $X_{\alpha}^{\text {ac }}$, where $q_{1}\left(q_{2}\right.$, resp. $)$ is a common denominator of the finite set of rational points of $\left(\mathbb{T}^{2} \times\{0\}\right) \cap X_{\alpha}^{\mathrm{ac}}$ $\left(\left(\{0\} \times \mathbb{T}^{2}\right) \cap X_{\alpha}^{\mathrm{ac}}\right.$, resp. $)$, we can assume that the intersection of $X_{\alpha}^{\text {ac }}$ with the " $x$ and $y$ axes" is empty, that is,

$$
X_{\alpha}^{\mathrm{ac}} \cap\left(\{0\} \times \mathbb{T}^{2} \cup \mathbb{T}^{2} \times\{0\}\right)=\emptyset .
$$


Suppose that $(0,0)$ is not isolated in $X_{\alpha}^{\mathrm{ac}}$. Thus there exists a sequence $\left\{\left(x_{n}, y_{n}\right)\right\} \subset X_{\alpha}^{\text {ac }}$ tending to $(0,0)$, with $x_{n}, y_{n} \neq 0$ by (5.11). Choosing an appropriate subsequence, we can assume that

$$
\lim _{n \rightarrow \infty} \frac{\left\|y_{n}\right\|}{\left\|x_{n}\right\|}=\alpha \in[0,+\infty) \quad \text { or } \quad \lim _{n \rightarrow \infty} \frac{\left\|y_{n}\right\|}{\left\|x_{n}\right\|}=+\infty,
$$

where $\|\cdot\|$ stands for an arbitrary norm in $\mathbb{R}^{2}$.

First we consider the case when $\alpha \neq 0$ or the limit in (5.12) is infinite. By the assumption (1.7) there are $k, l \in \mathbb{N}$ such that $m=m(k, l)$ has the property that

$$
\varrho_{2}^{\prime}>\varrho_{1}
$$

By (5.9) and (5.10) we get, for every $j \in \mathbb{N}$,

$$
\frac{\left\|m_{2}^{j} y_{n}\right\|_{2}}{\left\|m_{1}^{j} x_{n}\right\|_{1, \delta}} \geq\left(\frac{\varrho_{2}^{\prime}}{\varrho_{1}+\delta}\right)^{j} \frac{\left\|y_{n}\right\|_{2}}{\left\|x_{n}\right\|_{1, \delta}} .
$$

Now, by Lemma 5.4, we can choose a subsequence $v_{n_{k}}=\left(x_{n_{k}}, y_{n_{k}}\right)$ and $\left\{j_{n_{k}}\right\} \subset \mathbb{N}$ tending to infinity such that

$$
\lim _{k \rightarrow \infty} m_{2}^{j_{n_{k}}} y_{n_{k}}=y \neq 0 .
$$

By (5.13) we can take $\delta>0$ in (5.9) so that $\varrho_{2}^{\prime}>\varrho_{1}+\delta$. Hence, by (5.14) and our assumption that $\left\|y_{n_{k}}\right\|_{2} /\left\|x_{n_{k}}\right\|_{1, \delta}$ tends to $\alpha \neq 0$ or to infinity as $k \rightarrow \infty$,

$$
\lim _{k \rightarrow \infty} \frac{\left\|m_{2}^{j_{n_{k}}} y_{n_{k}}\right\|_{2}}{\left\|m_{1}^{j_{n_{k}}} x_{n_{k}}\right\|_{1, \delta}}=\infty .
$$

Now (5.15) and (5.16) imply that $m_{1}^{j_{n_{k}}} x_{n_{k}} \rightarrow 0$. Thus we have constructed the sequence $\left\{\left(m_{1}^{j_{n_{k}}} x_{n_{k}}, m_{2}^{j_{n_{k}}} y_{n_{k}}\right)\right\} \subset X_{\alpha}^{\text {ac }}$ such that $\left(m_{1}^{j_{n_{k}}} x_{n_{k}}, m_{2}^{j_{n_{k}}} y_{n_{k}}\right) \rightarrow$ $(0, y) \in \mathbb{T}^{2} \times \mathbb{T}^{2}$, with $y \neq 0$. This contradicts (5.11).

Finally, we consider the case when $\alpha=0$ in (5.12). By assumption (1.8) there are $k^{\prime}, l^{\prime} \in \mathbb{N}$ such that the corresponding element $m=m\left(k^{\prime}, l^{\prime}\right)$ in (5.8) satisfies $\varrho_{1}^{\prime}>\varrho_{2}$. By (5.9) and (5.10),

$$
\frac{\left\|m_{1}^{j} x_{n}\right\|_{1}}{\left\|m_{2}^{j} y_{n}\right\|_{2, \delta}} \geq\left(\frac{\varrho_{1}^{\prime}}{\varrho_{2}+\delta}\right)^{j} \frac{\left\|x_{n}\right\|_{1}}{\left\|y_{n}\right\|_{2, \delta}} .
$$

Now we proceed analogously to the previous case exchanging the roles of $x_{n}$ and $y_{n}$ to get a sequence $\left\{\left(m_{1}^{j_{n_{k}}} x_{n_{k}}, m_{2}^{j_{n_{k}}} y_{n_{k}}\right)\right\} \subset X_{\alpha}^{\text {ac }}$ such that, as $k \rightarrow \infty$, $\left(m_{1}^{j_{n}{ }_{k}} x_{n_{k}}, m_{2}^{j_{n_{k}}} y_{n_{k}}\right) \rightarrow(x, 0) \in \mathbb{T}^{2} \times \mathbb{T}^{2}$, with $x \neq 0$. This again contradicts (5.11).

COROLlary 5.17. With the same assumptions as in Theorem 1.6, either $(0,0)$ is isolated in $X_{\alpha}^{\mathrm{ac}}$, or $\left\{x+y:(x, y) \in X_{\alpha}^{\mathrm{ac}}\right\}=\mathbb{T}^{2}$. 


\section{Proof. Straightforward from Proposition 5.7.}

The following lemma will also be used. Its proof is analogous to the classical case of one endomorphism of $\mathbb{T}^{d}$ (see for example [11] or [1]). In this lemma $\Sigma \subset M_{\text {inv }}(d, \mathbb{Z}):=\mathrm{GL}(d, \mathbb{R}) \cap \mathrm{M}(d, \mathbb{Z})$, where $\mathrm{M}(d, \mathbb{Z})$ is the set of $d \times d$ matrices with integer entries, is a semigroup of endomorphisms of the $d$-dimensional torus $\mathbb{T}^{d}$. The torus $\mathbb{T}^{d}$ is endowed with its normalized Haar measure $m$, which is $\Sigma$-invariant.

Lemma 5.18. Assume $A \subset \mathbb{T}^{d}$ is measurable, has positive measure and satisfies $\Sigma A \subset A$. Then, if any character $\chi \neq \mathrm{Id}$ has unbounded $\Sigma^{t}$-orbit, then $A$ has measure 1 ; in particular $\Sigma$ is ergodic on $\mathbb{T}^{d}$.

Finally, we are ready to give

Proof of Theorem 1.6. Consider the set $X_{\alpha}, \alpha=\left(\alpha_{1}, \alpha_{2}\right)$, defined in (5.3), with $\alpha_{1}=\xi_{1}\left(1, \lambda_{1}\right)^{t}$ and $\alpha_{2}=\xi_{2}\left(1, \lambda_{2}\right)^{t}$ being common eigenvectors of the semigroups $\Sigma_{1}$ and $\Sigma_{2}$, respectively. We can assume that both $\xi_{1}$ and $\xi_{2}$ are non-zero; if one of them is zero then the conclusion of Theorem 1.6 follows from Theorem 1.3. Thus,

$$
X_{\alpha}=\left\{\left(\lambda_{1}^{n} \mu_{1}^{m} \xi_{1}, \lambda_{1}^{n+1} \mu_{1}^{m} \xi_{1}, \lambda_{2}^{n} \mu_{2}^{m} \xi_{2}, \lambda_{2}^{n+1} \mu_{2}^{m} \xi_{2}\right): n, m \in \mathbb{N}\right\} .
$$

We have noticed, before Lemma 5.4, that the semigroup $\Sigma_{1}=\left\langle\sigma_{1}, \tau_{1}\right\rangle$ satisfies the conditions of Theorem 3.1, and so is an ID-semigroup. Therefore, since $\alpha_{1}$ is not a rational point (see Remark 3.2), for every $x \in \mathbb{T}^{2}$ there exist sequences $\left\{n_{k}\right\}$ and $\left\{m_{k}\right\}$, tending to infinity, such that $\sigma_{1}^{n_{k}} \tau_{1}^{m_{k}} \alpha_{1} \rightarrow x$ as $k \rightarrow \infty$. Since $\mathbb{T}^{2}$ is compact, we can assume, choosing a subsequence, that $\sigma_{2}^{n_{k}} \tau_{2}^{m_{k}} \alpha_{2} \rightarrow y$ for some $y \in \mathbb{T}^{2}$. Therefore, for every $x \in \mathbb{T}^{2}$ there exists $y \in \mathbb{T}^{2}$ so that $(x, y) \in X_{\alpha}^{\text {ac }}$. In particular, $X_{\alpha}^{\text {ac }}$ is infinite.

By Lemma 4.3 there is a non-isolated rational point $\left(q_{1}, q_{2}\right)$ in $X_{\alpha}^{\text {ac }}$. For $\kappa, \iota \in \mathbb{N} \cup\{0\}$, define

$$
\mathcal{J}_{\kappa, \iota}=\left(\begin{array}{cc}
\operatorname{det} \sigma_{1} \mathrm{Id} & 0 \\
0 & \operatorname{det} \sigma_{2} \mathrm{Id}
\end{array}\right)^{\kappa}\left(\begin{array}{cc}
\operatorname{det} \tau_{1} \mathrm{Id} & 0 \\
0 & \operatorname{det} \tau_{2} \mathrm{Id}
\end{array}\right)^{\iota} .
$$

Let $\kappa, \iota$ be chosen so that, for $\left(\begin{array}{l}\widetilde{q}_{1} \\ \widetilde{q}_{2}\end{array}\right)=\mathcal{J}_{\kappa, \iota}\left(\begin{array}{c}q_{1} \\ q_{2}\end{array}\right)$, the denominator of $\widetilde{q}_{i}$ is relatively prime to $\operatorname{det} \sigma_{i}, \operatorname{det} \tau_{i}$ for $i=1,2$. Applying Lemma 4.4 to the set $\widetilde{X_{\alpha}^{\text {ac }}}=\mathcal{J}_{\kappa, L} X_{\alpha}^{\text {ac }}$ we can assume that $(0,0)$ is non-isolated in $\widetilde{X_{\alpha}^{\mathrm{ac}}}$. It is clear that Proposition 5.7 and Corollary 5.17 are valid for $\widetilde{X_{\alpha}^{\text {ac }}}$ instead of $X_{\alpha}^{\text {ac }}$, with no changes in their proofs. Thus, by Corollary 5.17, the set $\widetilde{S}:=\left\{x+y:(x, y) \in \widetilde{X_{\alpha}^{\text {ac }}}\right\}$ is equal to the whole $\mathbb{T}^{2}$. So its projection $\pi_{1}(\widetilde{S})$ of $\widetilde{S}$ on the first coordinate equals $\mathbb{T}$. But $\pi_{1}(\widetilde{S})=\left(\operatorname{det} \sigma_{1}\right)^{\kappa}\left(\operatorname{det} \tau_{1}\right)^{\iota} \pi_{1}(S)$, where $S=\left\{x+y:(x, y) \in X_{\alpha}^{\text {ac }}\right\}$. Thus we have

$$
\mathbb{T}=\left(\operatorname{det} \sigma_{1}\right)^{\kappa}\left(\operatorname{det} \tau_{1}\right)^{\iota} \pi_{1}(S) .
$$


Hence, a closed $\Sigma_{1}$-invariant subset $\pi_{1}(S)$ of $\mathbb{T}$ has positive Haar measure (greater than $\left.1 /\left(\operatorname{det} \sigma_{1}\right)^{\kappa}\left(\operatorname{det} \tau_{1}\right)^{\iota}\right)$. By Remark 5.2 and the condition on the eigenvalues of $\sigma_{1}$ and $\tau_{1}$, any character $\chi \neq \mathrm{Id}$ has unbounded $\Sigma^{t_{-}}$ orbit. Hence, by Lemma 5.18, the semigroup $\Sigma_{1}$ is ergodic. Thus $\pi_{1}(S)$ has measure 1. Since it is closed, we have $\pi_{1}(S)=\mathbb{T}$.

Now comparing this with the first and third coordinate of $X_{\alpha}$ in $\mathbb{T}^{2} \times \mathbb{T}^{2}$ (see (5.19)) we obtain the result.

Proof of Corollary 1.12. We slightly modify the proof of Theorem 1.6. The only difference is that when, say, $\lambda_{i}$ is of degree 1 , that is when $\lambda_{i}$ is an integer, we cannot define $\sigma_{\lambda_{i}}$ as in (5.1). Instead, if the corresponding $\mu_{i}$ is of degree 2 , we define

$$
\sigma_{\lambda_{i}}=\left(\begin{array}{cc}
\lambda_{i} & 0 \\
0 & \lambda_{i}
\end{array}\right)
$$

whereas if $\mu_{i}$ is of degree 1 , we define $\sigma_{\lambda_{i}}$ and $\tau_{\mu_{i}}$ as the $1 \times 1$ matrices,

$$
\sigma_{\lambda_{i}}=\left(\lambda_{i}\right) \text { and } \tau_{\mu_{i}}=\left(\mu_{i}\right) \text {. }
$$

For example, if we consider an expression as in (1.13), we have to deal with

$$
M_{\sigma}=\left(\begin{array}{ccc}
0 & 1 & 0 \\
-6 & 6 & 0 \\
0 & 0 & 2
\end{array}\right) \quad \text { and } \quad M_{\tau}=\left(\begin{array}{ccc}
5 & 0 & 0 \\
0 & 5 & 0 \\
0 & 0 & 7
\end{array}\right)
$$

acting on $\mathbb{T}^{2} \times \mathbb{T}$.

It is clear that Proposition 5.7 and Corollary 5.17, with obvious changes in their conclusions and some cosmetic changes in the proofs, work in this situation as well. In particular, in this example, Proposition 5.7 gives that, if $(0,0)$ is non-isolated in $\widetilde{X_{\alpha}^{\text {ac }}}$ then $\widetilde{X_{\alpha}^{\text {ac }}}$ contains $\mathbb{T}^{2} \times\{0\}$, where $0 \in \mathbb{T}$, or $\{0\} \times \mathbb{T}$, where $0 \in \mathbb{T}^{2}$. As in Corollary 5.17 we conclude that $\{\bar{x}+y:(x, y) \in$ $\left.\widetilde{X_{\alpha}^{\mathrm{ac}}}\right\}=\mathbb{T}$, where $\bar{x}$ is the projection of $x \in \mathbb{T}^{2}$ on the first coordinate.

\section{References}

[1] M. B. Bekka and M. Mayer, Ergodic Theory and Topological Dynamics of Group Actions on Homogeneous Spaces, London Math. Soc. Lecture Note Ser. 269, Cambridge Univ. Press, 2000.

[2] D. Berend, Multi-invariant sets on tori, Trans. Amer. Math. Soc. 280 (1983), 509532.

[3] -, Multi-invariant sets on compact abelian groups, ibid. 286 (1984), 505-535.

[4] - Dense $(\bmod 1)$ dilated semigroups of algebraic numbers, J. Number Theory 26 (1987), 246-256.

[5] M.-J. Bertin, A. Decomps-Guilloux, M. Grandet-Hugot, M. Pathiaux-Delefosse and J.-P. Schreiber, Pisot and Salem Numbers, with a preface by David W. Boyd, Birkhäuser, Basel, 1992. 
[6] M. D. Boshernitzan, Elementary proof of Furstenberg's Diophantine result, Proc. Amer. Math. Soc. 122 (1994), 67-70.

[7] H. Furstenberg, Disjointness in ergodic theory, minimal sets, and a problem in Diophantine approximation, Math. Systems Theory 1 (1967), 1-49.

[8] Y. Guivarc'h and A. N. Starkov, Orbits of linear group actions, random walk on homogeneous spaces, and toral automorphisms, Ergodic Theory Dynam. Systems 24 (2004), 767-802.

[9] Y. Guivarc'h and R. Urban, Semigroup actions on tori and stationary measures on projective spaces, Studia Math. 171 (2005), 33-66.

[10] G. H. Hardy and J. E. Littlewood, Some problems of Diophantine approximation, Acta Math. 37 (1914), 155-191.

[11] A. Katok and B. Hasselblatt, Introduction to the Modern Theory of Dynamical Systems, Encyclopedia Math. Appl. 54, Cambridge Univ. Press, Cambridge, 1995.

[12] B. Kra, A generalization of Furstenberg's Diophantine theorem, Proc. Amer. Math. Soc. 127 (1999), 1951-1956.

[13] L. Kuipers and H. Niederreiter, Uniform Distribution of Sequences, Pure Appl. Math., Wiley-Interscience, New York, 1974.

[14] D. Meiri, Entropy and uniform distribution of orbits in $\mathbb{T}^{d}$, Israel J. Math. 105 (1998), 155-183.

[15] Y. Meyer, Algebraic Numbers and Harmonic Analysis, North-Holland Math. Library 2, North-Holland, Amsterdam, 1972.

[16] R. Muchnik, Semigroup actions on $\mathbb{T}^{n}$, Geom. Dedicata 110 (2005), 1-47.

[17] W. A. Veech, Topological dynamics, Bull. Amer. Math. Soc. 83 (1977), 775-830.

Institute of Mathematics

Wrocław University

Plac Grunwaldzki 2/4

50-384 Wrocław, Poland

E-mail: urban@math.uni.wroc.pl

Received on 13.2.2006

and in revised form on 23.2.2007 